\title{
Corrigendum
}

\section{Acute D3 Antagonist GSK598809 Selectively Enhances Neural Response During Monetary Reward Anticipation in Drug and Alcohol Dependence}

Anna Murphy, Liam J Nestor, John McGonigle, Louise Paterson, Venkataramana Boyapati, Karen D Ersche, Remy Flechais, Shankar Kuchibatla, Antonio Metastasio, Csaba Orban, Filippo Passetti, Laurence Reed, Dana Smith, John Suckling, Eleanor Taylor, Trevor W Robbins, Anne Lingford-Hughes, David J Nutt, John FW Deakin, Rebecca Elliott and ICCAM Platform

Neuropsychopharmacology (2017) 42, 1559. doi:10.1038/npp.2017.47

Correction to: Neuropsychopharmacology advance online publication, 25 January 2017; doi:10.1038/npp.2016.289
This article was originally published under SN's License to Publish, but has now been made available under a CC BY 4.0 license. The PDF and HTML versions of the paper have been modified accordingly. 